\title{
Prevalence of Adverse Effects of COVID19 Vaccines among a Sample of Iranian Healthcare Workers; A Comparison between the Three Available Vaccines in Iran
}

\author{
Mohammad Mousavi ${ }^{1}$, Azita Tehranchi², Mahshid Namdari ${ }^{3}$, Maryam Sadeghipour ${ }^{*}$, Mohsen Dalband ${ }^{5}$, \\ Mahsa Malek Mohammadi ${ }^{3}$ Kazem Dalaie²
}

\begin{abstract}
'Dentofacial Deformities Research Center, Research Institute of Dental Sciences, Shahid Beheshti University of Medical Sciences, Tehran, Iran. 2Department of Orthodontics, School of Dentistry, Shahid Beheshti University of Medical Sciences, Tehran, Iran.

${ }^{3}$ Department of Community Oral Health, School of Dentistry, Shahid Beheshti University of Medical Sciences, Tehran, Iran.

${ }^{4}$ Department of Community Oral Health, Research Institute of Dental Sciences, Shahid Beheshti University of Medical Sciences, Tehran, Iran.

Department of Oral and Maxillofacial Surgery, School of Dentistry, Shahid Beheshti University of Medical Sciences, Tehran, Iran.

*Correspondence to: Maryam Sadeghipour (E-mail: Sadeghipour1393@gmail.com)
\end{abstract}

(Submitted: 29 September 2021 - Revised version received: 12 October 2021 - Accepted: 21 October 2021 - Published online: 26 December 2021)

\begin{abstract}
Objectives: Describing what to expect after the first and second dose of vaccination will increase the public confidence. This study aimed to describe the short-term side effects after receiving the first, the second, or both doses of Sputnik V, Oxford-AstraZeneca, and Sinopharm vaccines in personnel and students of shahid Beheshti dentistry school.

Methods: A cohort project has been conducting at the dental faculty since June 2020. This article is a cross-sectional study as a section of the cohort project. Dental faculty began its campaign to vaccinate the personnel on April 2021, with the Sputnik V and Oxford-AstraZeneca and Sinopharm. An employed operator phoned each one by one after 48 hours of vaccination. She asked them about any experienced symptoms after receiving each dose. The reported symptoms were coded and categorized.

Results: The most common symptoms in AstraZeneca's first dose were fever followed by Myalgia. After the second dose, the frequency of all symptoms had been reduced significantly. After the first dose of SputnikV, the most common symptoms were Myalgia followed by fever. The frequency of fever and pain at the injection site increased significantly after the second dose $(P<0.001)$. In the Sinopharm group, all symptoms occurred whit a low percentage. The most common symptoms were again, Myalgia and pain at the injection site.

Conclusion: Post-vaccination adverse effects were mild in all groups and controlled by analgesic. Fever, myalgia and pain at the injection site are the most common symptoms reported after vaccination by all three types of vaccines.

Keywords: Adverse effects, COVID-19, vaccine
\end{abstract}

\section{Introduction}

During the COVID-19 pandemic, people face significant health care challenges, lockdowns, anxiety, and stress, as there is no specific treatment for this pandemic. However, vaccine development is perhaps the best hope to stop this pandemic. ${ }^{1}$

The most effective vaccines mimic the preventive mechanism resulting from the recovery phase in the standard form of the disease. ${ }^{2}$ Herd immunity is established when sufficient people are immune to stop new cases, which means that enough people are protected to stop person-to-person transmission in the community.

The percentage of people who need to achieve herd immunity varies with specific diseases, e.g., measles needs about 95\% of vaccination among the community. To achieve herd immunity for COIVID-19, the exact percentage is not known.

Since the outbreak, multiple vaccine candidates based on RNA and DNA technologies inactivated viruses, and other approaches have been rapidly developed. Doroftei et al. concluded that three vaccines had more than $90 \%$ efficacy (PfizerBioNTech, 95\%; Moderna, 94\%; and Sputnik V, 92\%) except for Oxford-AstraZeneca $(\sim 81 \%)$.

Iran's drug regulatory authority has given emergency approval to four vaccines for restricted use against covid-19, including Sputnik V, COVAX in, Sinopharm, and AstraZeneca. Many health workers in the dentistry school of Shahid Beheshti University have welcomed the program and undergone vaccination with Sputnik V, Oxford-AstraZeneca (ChAdOx1 nCoV-19), and Sinopharm since March 2021.

Sputnik V has been used in many countries. The adenoviruses are combined with the SARS-CoV-2 spike protein to make the vaccine, which prompts the body to make an immune response. Interim results (based on data from 14,964 participants in the vaccine group and 4902 in the placebo group) indicate that the vaccine is $91.6 \%$ effective, based on its ability to prevent symptomatic infection. There were no cases of moderate or severe covid-19 in the vaccinated group at least 21 days following the first dose. No serious adverse events were detected. Most adverse effects were mild, with over half experiencing pain at the injection site. ${ }^{6}$

Oxford-AstraZeneca is a viral vector vaccine using a modified chimpanzee adenovirus ChAdOx $1 .{ }^{7}$ The vaccine's efficacy is $76.0 \%$ in preventing symptomatic COVID-19 in 22 days following the first dose and $81.3 \%$ after the second. The vaccine has a good safety profile, with side effects including injection-site pain, headache, and nausea, generally resolving within a few days. More rarely, anaphylaxis may occur. In rare cases (around 1 in 100,000), the vaccine has been associated with an increased risk of blood clots in combination with low blood platelet level. ${ }^{8}$

As with any other medicine, vaccines are not free from side effects and adverse reactions, most of which are generally mild, well-tolerated, and self-resolving. These symptoms could indicate the body is developing the desired immunity. ${ }^{9}$ 
Fear of the new vaccines is a driver of vaccine hesitancy. ${ }^{10}$ The knowledge about what happens post-vaccination in the actual world among the general population is still modest, Thus, describing what to expect after the first and second dose of vaccination will help in lowering the apprehension about this type of vaccines, increased and also increases the public confidence and safety, and accelerates the vaccination process against COVID-19.

This study aimed to describe the short-term side effects after receiving the first, the second, or both doses of Sputnik V, Oxford-AstraZeneca, and Sinopharm COVID-19 vaccines in a sample of 18 years and older personnel and students of shahid Beheshti dentistry school.

\section{Materials and Methods}

\section{Study Design and Participants}

A comprehensive cohort project has been conducting at the dental faculty of Shahid Beheshti University of medical sciences since the reopening of universities in Iran in June 2020. All personnel were screened and followed. Contraction to the Covid-19, Signs, and symptoms after contraction, systemic condition, types of activities, and precautional behaviors have been monitored and registered. This article is a crosssectional study as a section of the cohort project. The present study was conducted over the period between April 10 to August 1, 2021. Ethical approval was taken from Shahid Beheshti University of medical sciences' review committee.

\section{Procedures}

According to CDC and WHO protocols, the dental faculty began its campaign to vaccinate the personnel against the emerging coronavirus on April 2021, with the Sputnik V and Oxford-AstraZeneca and Sinopharm. All the students and staff were vaccinated at the same time. Types of vaccines with the date of injection of first and second dose were summarized in Table 1. 176 doses of Oxford-AstraZeneca and 600 doses of Sputnik V were used in the vaccination.

Informed consent was obtained from each individual who was rejected to be vaccinated based on WHO advice.

A resident general practitioner was established at the vaccination site to inform and observe any adverse effects. Following the vaccination, all recipients were observed for 30 minutes.

Probable occurrence of side effects and adverse effects following immunization (AEFI) like Injection site pain, swelling, redness; fever, chills, rigor, sweating, headache, muscle ache, joint pain, cough, running nose, sore throat, diarrhea, nausea, vomiting, abdominal cramps, loss of appetite, skin rashes, itching, palpitation, dizziness, fainting, shortness of breath, chest tightness, was explained to each participant. A phone number was given to them to report any experienced adverse effects, including both systemic (whole-body) and local effects, following 30 days after receiving each dose.

An employed operator phoned each one by one after 48 hours of vaccination. She asked them about any experienced symptoms after receiving the first/second dose and asked them if any medicines or painkillers were taking during those days.

The reported symptoms were coded and categorized as shown in Table 1.

\begin{tabular}{|c|c|}
\hline Code & Symptoms \\
\hline 1 & Fever/chilling \\
\hline 2 & Headache/dizziness \\
\hline 3 & Myalgia/backache/weakness/fatigue/Lethargy \\
\hline 4 & gastrointestinal \\
\hline 5 & Tachycardia/hypoxemia/Heart pain/cough \\
\hline 6 & Pain/swelling at injection site \\
\hline 7 & Flu-like symptoms (rhinitis etc.) \\
\hline
\end{tabular}

Table 2. Total number of vaccinated individuals in each vaccination group and distribution of genders

\begin{tabular}{lccc}
\hline Vaccine type & $\begin{array}{c}\text { Male } \\
\text { (percentage) }\end{array}$ & $\begin{array}{c}\text { Female } \\
\text { (percentage) }\end{array}$ & $\begin{array}{c}\text { Total } \\
\text { number }\end{array}$ \\
\hline AstraZeneca & $58.3 \%$ & $41.7 \%$ & 192 \\
SputnikV & $44.5 \%$ & $55.5 \%$ & 236 \\
Sinopharm & $47.7 \%$ & $52.3 \%$ & 44 \\
\hline
\end{tabular}

\section{Statistical Analysis}

IBM-SPSS statistical package for social sciences version 21 (IBM Corp: Armonk, NY, USA) was used to obtain descriptive statistics and significant associations between variables. Descriptive statistics were reported as frequencies and percentages. Cross tabulation with McNemar's test was carried out to compare the frequency of individuals with positive side effects after each dose in each vaccine type. A Chi-square test was used to compare the frequency of side effects after the first and second doses between different vaccine types.

\section{Results}

The total number of vaccinated individuals in each vaccination group and distribution of genders were summarized in Table 2. From 472 personnel and students, 236 were vaccinated with sputnik V while 192 received AstraZeneca and 44 received Sinopharm's first dose.

Side effects of each vaccine in every dose were summarized in Table 3.

The most common symptoms in AstraZeneca's first dose were fever followed by Myalgia. 87.2\% of individuals who received medicine after the first dose. After the second dose, the frequency of all symptoms had been reduced significantly except tachycardia, cough, and flu-like symptoms. $(P<0.001)$ Indeed, the frequency of those who had such symptoms in the first dose was significantly more than individuals who had the symptoms only after the second dose. However, the percentage of taking medication reduced significantly; still, $51.1 \%$ of participants used drugs after the second dose.

After the first administration dose, the most common symptoms were Myalgia followed by fever for the Sputnik V group. $57.3 \%$ of individuals received medicine after the first dose. After the second dose, the frequency of fever and pain at the injection site increased significantly. The percentage of individuals who reported fever and pain at the injection site only after the second dose was significantly more than those who reported fever and pain only after the first dose. 
$(P<0.001) 57.1$ of participants received medicine to relieve pain after the second dose.

In the Sinopharm group, all symptoms occurred whit a low percentage. The most common symptoms after the first dose were, again, Myalgia and pain at the injection site. Nobody reported gastrointestinal problems or Tachycardia/ hypoxemia/Heart pain/cough. Among all participants, gastrointestinal symptoms or flu-like symptoms were reported at least.

After the first dose injection, the results showed a significant difference in the report of side effects among individuals vaccinated with different vaccines except for tachycardia and flu-like symptoms $(P$ value $<0.05)$ (Figure 1$)$.

After the injection of the second dose of AstraZeneca and Sputnik V, the report of fever, Myalgia, and pain at the injection site were significantly different between the two types of vaccines $(P=0.0)$ (Figure 2$)$.

\section{Discussion}

There is a variation in people's acceptance to take the vaccines due to several factors. An important factor may be the shorttime development of new vaccines compared to the previously approved vaccines. Another reason for this variation may be the usage of a newly emerging technique for mRNA vaccines. There was also exaggeration and over-reporting of adverse effects of vaccines, as some of these effects are normal physiologic processes or developmental anomalies. ${ }^{11-13}$ Thus, this study aimed to evaluate the short-term side effects of COVID-19 vaccines, which are currently used in Shahid Beheshti Dental school.

In this study, these systemic symptoms, including Myalgia, backache, weakness, fatigue, and Lethargy, were the most commonly reported symptoms after the injection of the first dose of each vaccine. The second common symptom in AstraZeneca and sputnik groups was fever in each dose. However,

\begin{tabular}{|c|c|c|c|c|c|}
\hline \multirow[t]{2}{*}{ Side effects/taking medication } & \multicolumn{2}{|c|}{ AstraZeneca } & \multicolumn{2}{|c|}{ Sputnik } & \multirow{2}{*}{$\begin{array}{c}\text { Sinopharm } \\
\begin{array}{c}\text { First dose } \\
\text { (percentage) }\end{array}\end{array}$} \\
\hline & $\begin{array}{c}\text { First dose } \\
\text { (percentage) }\end{array}$ & $\begin{array}{l}\text { Second dose } \\
\text { (percentage) }\end{array}$ & $\begin{array}{l}\text { First dose } \\
\text { (percentage) }\end{array}$ & $\begin{array}{l}\text { Second dose } \\
\text { (percentage) }\end{array}$ & \\
\hline Fever/chilling & 70.2 & $25.1^{*}$ & 38.5 & $46.6^{*}$ & 8.3 \\
\hline Headache/dizziness & 28.7 & $7.5^{*}$ & 11.8 & 13.6 & 5.6 \\
\hline Myalgia/back ache weakness/fatigue/Lethargy & 63.5 & $23.5^{*}$ & 46.4 & 45.2 & 25.0 \\
\hline Gastrointestinal & 11.7 & $2.9^{*}$ & 3.6 & 5.5 & 0 \\
\hline Tachycardia/hypoxemia/Heart pain/cough & 3.5 & 6 & .9 & 1.8 & 0 \\
\hline Pain/swelling at injection site & 14.9 & $3.4^{*}$ & 2.3 & $8.6^{*}$ & 19.4 \\
\hline Flu-like symptoms (rhinitis etc.) & 1.6 & 1.1 & 3.6 & 1.4 & 2.8 \\
\hline Taking medication & 87.2 & $51.1^{*}$ & 57.3 & 57.1 & 19.4 \\
\hline
\end{tabular}

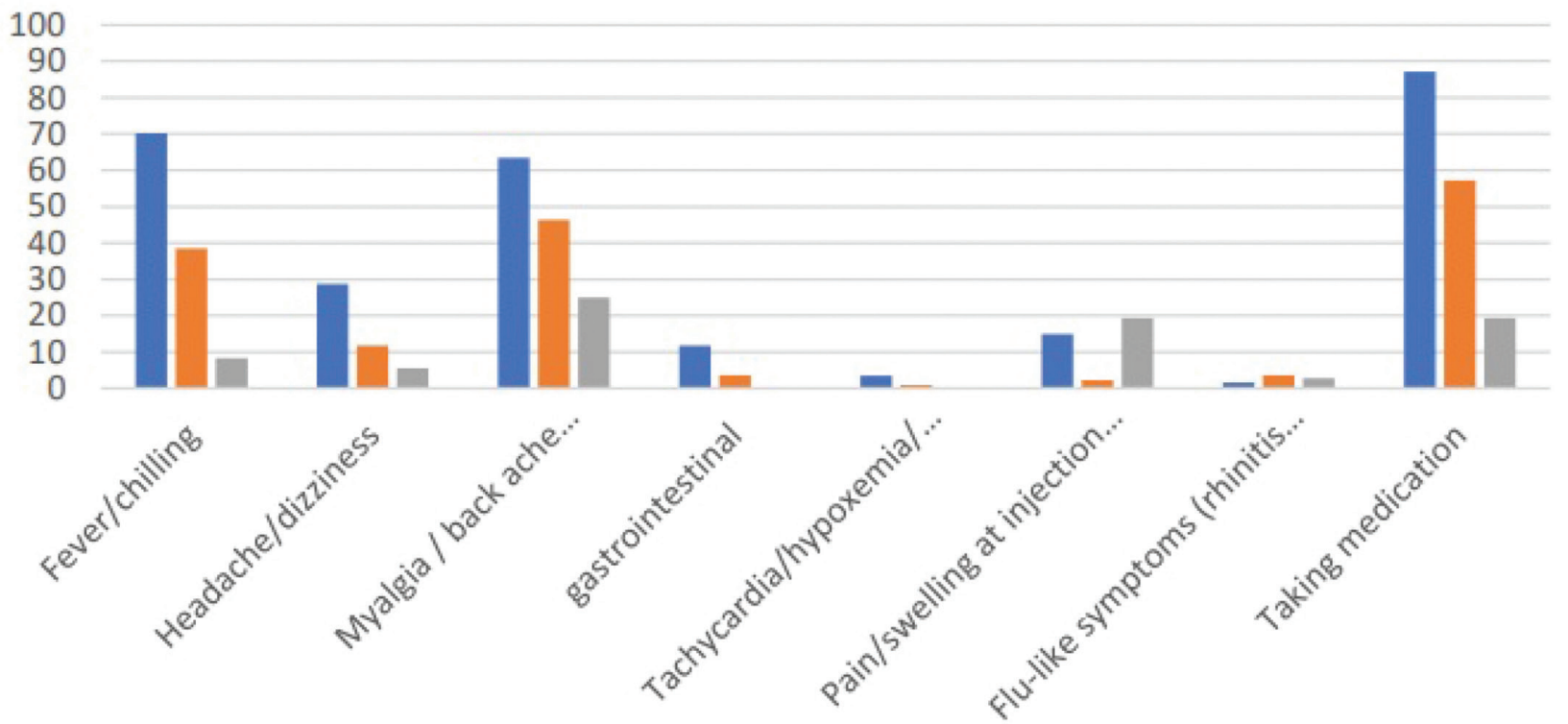

AstraZeneca Sputnik $\quad$ Sinopharm

Fig. 1 Comparison of side effects after the injection of first dose between vaccines. 


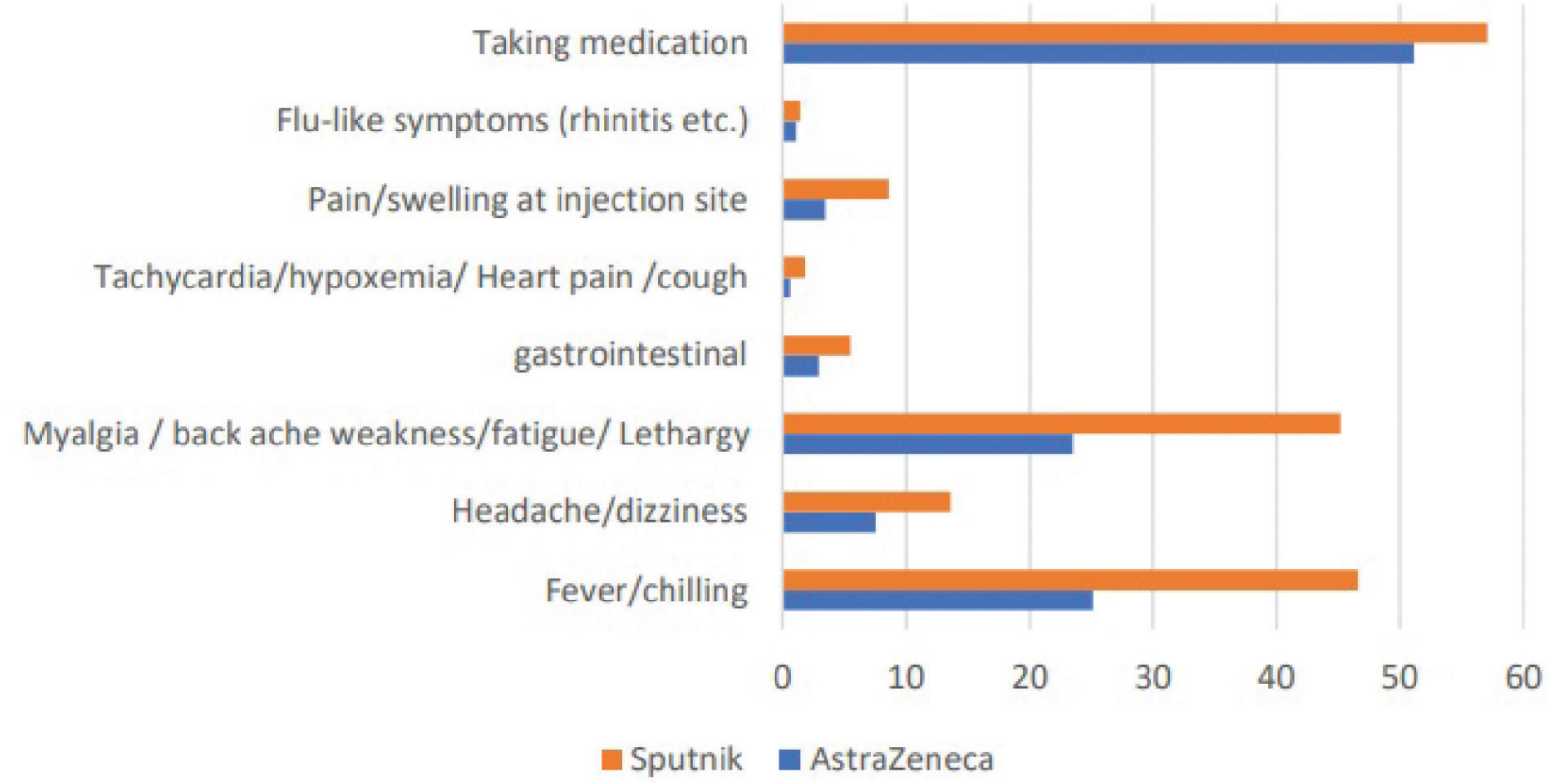

Fig. 2 Comparison of side effects after the injection of second dose between vaccines.

in the Sinopharm group, the second common symptom was local pain at the injection site. All these were mild and selflimiting. Moreover, no treatment was required for any side effects. Most similar cross-sectional studies ${ }^{4,10,14,15}$ also reported fever, tiredness, and pain at the injection site as the most routine symptoms after vaccination.

Not surprisingly, fever was one of the most frequently reported adverse reactions. Similar evidence has been earlier garnered from the phase III clinical trials of some of these vaccines, where the highest frequency of fever was up to $24 \%$ with AstraZeneca Vaxzervria. ${ }^{4}$

Systemic symptoms are that the immune system could produce cytokines that affect the blood vessels, muscles, and other tissues. It may also produce flu-like symptoms that last for days after vaccination. ${ }^{16}$

Alhazmi et al. reported that most of the participants in their study had tiredness and headache, and they stated that this was mainly due to the younger age of our participants. Similarly, in this study, participants were young or below the retirement age. ${ }^{12}$

Vaccines in "in situ" should be kept at a low temperature, including Sinopharm vaccine that should store at average refrigeration temperature, and if injected without optimal warming up, this may increase the probability of pain of the injection site symptom.

Although all COVID-19 vaccines cause similar postvaccination side effects, the severity and number of these side effects were significantly associated with vaccine type. In this study, AstraZeneca caused more side effects after the first dose than Sputnik V and Sinopharm. ${ }^{15}$ Similarly, Menni et al. reported that those vaccinated with the ChAdOx1 nCoV-19 vaccine were more likely to experience systemic side effects than those who had been given other types. ${ }^{17}$

Several viral vaccine development platforms include live attenuated, inactivated, DNA-based, RNA-based, proteinbased, and viral vector-based.

AstraZeneca vaccine consists of ChAdOxl (replicationdeficient simian adenovirus vector) containing the full-length structural SARS-CoV-2 spike (S) protein. The $S$ protein plays a crucial role in penetrating host cells and initiating infection of all SARS viruses. The AstraZeneca vaccine expresses a codon-optimized coding sequence for the $S$ protein in the human body, which builds immunity against SARS-CoV-2. ${ }^{18}$

Sputnik $\mathrm{V}$ is a recombinant vaccine using human adenovirus vector 26 for the first vaccine and human adenovirus 5 for the second vaccine. ${ }^{2}$

The Sinopharm vaccine has been developed using conventional technology (i.e., inactivated virus). DNA vaccines are easy to produce and store with excellent stability and limited toxicity. ${ }^{19,20}$ The results of Abu-Hammad et al. study also study confirmed that Sinopharm is a "quiet" vaccine since it was significantly associated with symptom-free vaccination. ${ }^{13}$

The results showed that almost all post-vaccination symptoms in the AstraZeneca group, except tachycardia and flu-like symptoms, occurred more frequently after the first dose than the second dose. $(P<0.001)$.

In the Sputnik V group, side effects were more noticeable after the second dose. However, the frequency of only fever and pain in the injection site was significant, and most side effects showed no significant association with the number of doses.

In a similar study, Hatmal et al. assessed the side effects of Sinopharm, AstraZeneca, Pfizer-BioNTech in Jordan, and they also reported that side effects after the first dose were more intense than the second. ${ }^{15}$ However, according to the Centers for Disease Control and Prevention (CDC), side effects might be more noticeable after the second dose. ${ }^{21,22}$ It seems that in individuals taking the AstraZeneca vaccine, the immune system will be activated more vigorously after the first dose.

In this study, participants reported taking medication to alleviate pain and reduce symptoms. The percentage of individuals who received medicine was $87.2 \%$ for AstraZeneca's first dose to $19.4 \%$ for Sinopharm's first dose. In the AstraZeneca group, there was a significant association between taking medication and the number of doses. AstraZeneca 
Company stated that prophylactic use of Acetaminophen could reduce some symptoms. ${ }^{2}$

Based on the results, the percentage of taking medications after both doses of Sputnik V was similar to the second dose of AstraZeneca. Although, side effects after the second dose of AstraZeneca had been reduced. Previous studies suggested non-steroidal anti-inflammatory drugs to control fever or antihistamines for topical side effects of the Sputnik V vaccine., ${ }^{2,15}$

Such mild side effects are acceptable during COVID-19 vaccination as the body will need some time to adopt vaccination doses and to trigger the immune system. The population should be informed about these minor side effects controlled with some symptomatic treatment like paracetamol. Such medicine should also be taken as prophylaxis to avoid developing symptoms and increase the acceptance of the COVID-19 vaccine among the mass population. It will help to counter this pandemic disease through ongoing vaccination program successfully. ${ }^{14}$

The strengths of the study include its large sample size and diversity of vaccines. This study also has some limitations. Self-reported data were used, which can introduce information bias. Also, the second dose of vaccination with Sinopharm has not been completed. Since this study was a part of a comprehensive cohort study and all personnel and student were counted, the sex and age of participants could not be matched for the results. It is suggested for future studies to evaluate objectively the antibody response to each vaccine administrated against covid 19 besides subjective reports.

\section{Conclusion}

Our study confirmed that post-vaccination adverse effects were mild in all groups and controlled by analgesic. In AstraZeneca group, systemic reactions were more intense and occurred after the first dose more frequently compared with other groups. In general, Fever, myalgia and pain at the injection site are the most common symptoms reported after vaccination by all three types of vaccines.

\section{Conflicts of Interest}

None.

\section{References}

1. Meo S, Bukhari I, Akram J, Meo A, Klonoff D. COVID-19 vaccines: comparison of biological, pharmacological characteristics and adverse effects of Pfizer/BioNTech and Moderna Vaccines. Eur Rev Med Pharmacol Sci. 2021;25(3):1663-9.

2. Ghiasi N, Arabsorkhi M, Hoseyni T, Esfandiari K, Sadighpour T, Jahantigh H. Efficacy and side effects of SputnikV, Sinopharm and AstraZeneca vaccines to stop COVID-19; a review and discussion. Immunopathologia Persa. 2021;7(2):31.

3. Metcalf CJE, Ferrari M, Graham AL, Grenfell BT. Understanding herd immunity. Trends in immunology. 2015;36(12):753-5.

4. Lippi G, Mattiuzzi C, Henry BM. Mild Adverse Reactions after COVID-19 Vaccination: Updated Analysis of Italian Medicines Agency Data. Available at SSRN 3817988. 2021

5. Doroftei B, Ciobica A, Ilie O-D, Maftei R, Ilea C. Mini-review discussing the reliability and efficiency of COVID-19 vaccines. Diagnostics. 2021;11(4):579.

6. Baraniuk C. Covid-19: What do we know about SputnikV and other Russian vaccines? bmj. 2021;372.

7. Raadsen M, Du Toit J, Langerak T, van Bussel B, van Gorp E, Goeijenbier M. Thrombocytopenia in virus infections. Journal of Clinical Medicine. 2021;10(4):877

8. Ramasamy MN, Minassian AM, Ewer KJ, Flaxman AL, Folegatti PM, Owens $\mathrm{DR}$, et al. Safety and immunogenicity of ChAdOx $1 \mathrm{nCoV}-19$ vaccine administered in a prime-boost regimen in young and old adults (COV002): a single-blind, randomised, controlled, phase 2/3 trial. The Lancet. 2020:396(10267):1979-93

9. Song BJ, Katial RK. Update on side effects from common vaccines. Current allergy and asthma reports. 2004:4(6):447-53.

10. Saeed BQ, Al-Shahrabi R, Alhaj SS, Alkokhardi ZM, Adrees AO. Side effects and perceptions following Sinopharm COVID-19 vaccination. International Journal of Infectious Diseases. 2021:111:219-26.

11. El-Shitany NA, Harakeh S, Badr-Eldin SM, Bagher AM, Eid B, Almukadi H, et al. Minor to moderate side effects of Pfizer-BioNTech COVID-19 vaccine among Saudi residents: A retrospective cross-sectional study. International journal of general medicine. 2021;14:1389.
12. Alhazmi A, Alamer E, Daws D, Hakami M, Darraj M, Abdelwahab S, et al. Evaluation of Side Effects Associated with COVID-19 Vaccines in Saudi Arabia. Vaccines. 2021;9(6):674

13. Abu-Hammad O, Alduraidi H, Abu-Hammad S, Alnazzawi A, Babkair H, Abu-Hammad A, et al. Side Effects Reported by Jordanian Healthcare Workers Who Received COVID-19 Vaccines. Vaccines. 2021;9(6):577.

14. Joshi U, Singh P. Adverse reaction following COVISHEILD a covid 19 vaccine-Experience of from a single Centre.

15. Hatmal MmM, Al-Hatamleh MA, Olaimat AN, Hatmal M, Alhaj-Qasem DM, Olaimat TM, et al. Side Effects and Perceptions Following COVID-19 Vaccination in Jordan: A Randomized, Cross-Sectional Study Implementing Machine Learning for Predicting Severity of Side Effects. Vaccines. 2021;9(6):556.

16. Hervé C, Laupèze B, Del Giudice G, Didierlaurent AM, Da Silva FT. The how's and what's of vaccine reactogenicity. npj Vaccines. 2019:4(1):1-11.

17. Menni C, Klaser K, May A, Polidori L, Capdevila J, Louca P, et al. Vaccine after Effects and Post-Vaccine Infection in a Real World Setting: Results from the COVID Symptom Study App. 2021.

18. Folegatti PM, Ewer KJ, Aley PK, Angus B, Becker S, Belij-Rammerstorfer S, et al. Safety and immunogenicity of the ChAdOx1 nCoV-19 vaccine against SARS-CoV-2: a preliminary report of a phase 1/2, single-blind, randomised controlled trial. The Lancet. 2020;396(10249): 467-78.

19. Silveira MM, Moreira GMSG, Mendonça M. DNA vaccines against COVID-19: Perspectives and challenges. Life sciences. 2020:118919.

20. Abbood, Muhanad Hadi; HASSAN, Jabbar Sirhan; MEHAMMED, Aqeel Jassim. Case study of COVID 19 from August to end of December 2020 in Babylon, Iraq. Iraq Medical Journal, v. 5, n. 3, sep. 2021

21. Control CfD, Prevention. Possible side effects after getting a COVID-19 vaccine. 2021.

22. Al-khayat, Zakarea Abdullah Yaseen et al. Single Center Study of Vaccination Breakthrough infection with SARS-CoV-2 Among Erbil Population in August 2021. Journal of Contemporary Medical Sciences, v. 7, n. 5, oct. 2021. 